\title{
N-DIMENSIONS LINEAR THERMOELASTIC SYSTEM IN DOMAINS WITH MOVING BOUNDARY
}

\author{
Cruz S. Q. De Caldas*, \\ Juan Limaco, \\ Rioco K. Barreto
}

\begin{abstract}
In this article the authors investigated the existence of solutions for the linear thermoelastic system in a noncylindrical domain $\hat{Q}$ of $\mathfrak{R}^{n+1}$.
\end{abstract}

\section{Introduction}

Let $K:[0, T] \rightarrow \Re$ be a $C^{2}$ function and let $\Omega$ be an open bounded set in $\Re^{n}$, with regular boundary. Let us consider the family of open sets in $\mathfrak{R}^{n+1}$ :

$$
\Omega_{t}=\{K(t) y ; \quad y \in \Omega\}, \quad 0 \leq t \leq T,
$$

with lateral boundary $\Gamma_{t}$. We define the noncylindrical domain $\hat{Q}$ in $\mathfrak{R}^{n+1}$ by:

$$
Q=\bigcup_{0<t<T} \Omega_{t} \times\{t\}
$$

with lateral boundary

$$
\Sigma=\bigcup_{0<t<T} \Gamma_{t} \times\{t\} .
$$

In this work we investigate the existence of solutions for the following thermoelastic system in $\hat{Q}$ :

$$
\mid \begin{aligned}
& u^{\prime \prime}-\mu \Delta u-(\lambda+\mu) \nabla \operatorname{div} u+\alpha \nabla \theta=0 \text { in } \hat{Q} \\
& \theta^{\prime}-\Delta \theta+\beta \text { div } u_{t}=0 \text { in } \hat{Q} \\
& u=0, \theta=0 \text { in } \hat{\Sigma} \\
& u(x, 0)=u_{0}(x), u^{\prime}(x, 0)=u_{1}(x), \theta(x, 0)=\theta_{0}(x) \text { in } \Omega_{0} .
\end{aligned}
$$

* Departamento de Matemática Aplicada - IMUFF, Universidade Federal Fluminense, Rua Mário Santos Braga s/n², 24020-140 Niterói, RJ, Brasil.E-mail: gmacruz@vm.uff.br 
We denote by $x=\left(x_{1}, \ldots, x_{n}\right)$ a point in $\Re^{n}$ while $t$ stands for the time variable. The displacement-vector is denoted by $u(x, t)=\left(u_{1}(x, t), \ldots, u_{n}(x, t)\right)$ and the thermal difference by $\theta=\theta(x, t) ; \lambda, \mu>0$ are Lemé's constants and $\alpha, \beta>0$ the coupling parameters.

There is an extensive literature for the study of $\left(^{*}\right)$ in cylindrical domains. It can be seen in [1], [3], [4], [5] and [6]. For the one-dimensional noncylindrical case, in [2], it is studied the existence, uniqueness and energy decay of weak solutions. We don't know results for (*) in n-dimensional case in noncylindrical domains.

\section{Notations and main Results}

Let us consider the real function $K(t)$ satisfying to the following conditions:

H1) $K \in C^{3}([0, T] ; \Re)$ with $K_{0}=\min _{0 \leq t \leq T} K(t)>0$.

H2) There exists a positive constant $K_{1}$, such that

$$
\left[\mu \delta_{i}^{j}-\left(K^{\prime}\right)^{2} y_{i} y_{j}\right] \in_{i} \in_{j} \geq K_{1}|\varepsilon|_{\mathfrak{R}^{n}}^{2} \quad \forall \varepsilon \in \mathfrak{R}^{n}, \forall y \in \Omega, \quad 0 \leq t \leq T .
$$

Remark. It is noted that when $(x, t)$ varies in $\hat{Q}$ the point $(y, t)$ with $y=K^{-1}(t) x$ varies in the cylinder $Q=\Omega \times] 0, T[$. Thus, the application

$$
\tau: \hat{Q} \rightarrow Q
$$

given by $\tau(x, t)=(y, t)$, is a diffeomorfism. Let us consider the change of variable in $\left(^{*}\right)$, given by

$$
\begin{gathered}
v(y, t)=u(K(t) y, t) \\
\phi(y, t)=\theta(K(t) y, t),
\end{gathered}
$$

We obtain the following mixed problem:

$$
\mid \begin{gathered}
v^{\prime \prime}-\frac{\partial}{\partial y_{j}}\left[a_{i j}(y, t) \frac{\partial v}{\partial y_{i}}\right]-\frac{(\lambda+\mu)}{K^{2}} \nabla \operatorname{div} v+\frac{\alpha}{K} \nabla \phi+b_{i}(y, t) \frac{\partial v^{\prime}}{\partial y_{i}} \\
+c_{i}(y, t) \frac{\partial v}{\partial y_{i}}=0 \quad \text { in } Q \\
\phi^{\prime}-\frac{1}{K^{2}} \Delta \phi+\frac{\beta}{K} d i v v^{\prime}+d_{i}(y, t) \frac{\partial \phi}{\partial y_{i}}+\frac{\beta K^{\prime}}{K^{2}} \operatorname{div} v \\
+e_{i}(y, t) \frac{\partial^{2} v_{j}}{\partial y_{j} \partial y_{i}}=0 \quad \text { in } \quad Q \\
\begin{array}{l}
v=0, \phi=0 \quad \text { in } \quad \Sigma \\
v(y, 0)=v_{0}(y), v^{\prime}(y, 0)=v_{1}(y), \phi(y, 0)=\phi_{0}(y), \quad y \in \Omega
\end{array}
\end{gathered}
$$


where

$$
\begin{aligned}
& a_{i j}(y, t)=\left[\mu \delta_{i}^{j}-\left(K^{\prime}\right)^{2} y_{i} y_{j}\right] K^{-2}, \quad b_{i}(y, t)=\frac{-2 K^{\prime}}{K} y_{i} \\
& c_{i}(y, t)=\left[(1-n)\left(K^{\prime}\right)^{2}-K^{\prime \prime} K\right] K^{-2} y_{i}, \quad d_{i}(y, t)=\frac{-y_{i} K^{\prime}}{K}, \quad e_{i}(y, t)=\frac{-\beta y_{i} K^{\prime}}{K^{2}} .
\end{aligned}
$$

Let us represent by $(()),.\|\cdot\|$ and $(),.|\cdot|$, the scalar product and norm in $\left[H_{0}^{1}(\Omega)\right]^{n}$ and $\left[L^{2}(\Omega)\right]^{n}$ respectively. We denote by $a(t, v, w)$ the bilinear form defined in $\left[H_{0}^{1}(\Omega)\right]^{n}$ by:

$$
a(t, v, w)=\int_{\Omega} a_{i j}(y, t) \frac{\partial v}{\partial y_{i}} \cdot \frac{\partial w}{\partial y_{j}} d y
$$

Note that $\operatorname{ax}(t, v, w)$ is continuous, symmetric and coercive.

The main results in our work are:

Teorema 1. Given $u_{0} \in\left[H_{0}^{1}\left(\Omega_{0}\right) \cap H^{2}\left(\Omega_{0}\right)\right]^{n}, \theta_{0} \in H_{0}^{1}\left(\Omega_{0}\right) \cap H^{2}\left(\Omega_{0}\right)$, and $u_{1} \in\left[H_{0}^{1}\left(\Omega_{0}\right)\right]^{n}$, then there exist functions

$$
u: \hat{Q} \rightarrow \Re^{n} \text { and } \theta: \hat{Q} \rightarrow \Re
$$

such that:

$$
\begin{aligned}
& u \in L^{\infty}\left(0, T ;\left[H_{0}^{1}\left(\Omega_{t}\right) \cap H^{2}\left(\Omega_{t}\right)\right]^{n}\right), u^{\prime} \in L^{\infty}\left(0, T ;\left[H_{0}^{1}\left(\Omega_{t}\right)\right]^{n}\right), u^{\prime \prime} \in L^{\infty}\left(0, T ;\left[L^{2}\left(\Omega_{t}\right)\right]^{n}\right) \\
& \theta \in L^{2}\left(0, T ; H_{0}^{1}\left(\Omega_{t}\right) \cap H^{2}\left(\Omega_{t}\right)\right), \theta^{\prime} \in L^{2}\left(0, T ; H_{0}^{1}\left(\Omega_{t}\right)\right),
\end{aligned}
$$

and they are solutions of the problem $\left(^{*}\right)$ in $\hat{Q}$.

Teorema 2. Given $v_{0} \in\left[H_{0}^{1}(\Omega) \cap H^{2}(\Omega)\right]^{n}, \phi_{0} \in H_{0}^{1}(\Omega) \cap H^{2}(\Omega)$, and $v_{1} \in\left[H_{0}^{1}(\Omega)\right]^{n}$, then there exist functions

$$
v: Q \rightarrow \mathfrak{R}^{n} \text { and } \phi: Q \rightarrow \mathfrak{R}
$$

such that:

$$
\begin{aligned}
& v \in L^{\infty}\left(0, T ;\left[H_{0}^{1}(\Omega) \cap H^{2}(\Omega)\right]^{n}\right), v^{\prime} \in L^{\infty}\left(0, T ;\left[H_{0}^{1}(\Omega)\right]^{n}\right), v^{\prime \prime} \in L^{\infty}\left(0, T ;\left[L^{2}(\Omega)\right]^{n}\right) \\
& \phi \in L^{2}\left(0, T ; H_{0}^{1}(\Omega) \cap H^{2}(\Omega)\right), \phi^{\prime} \in L^{2}\left(0, T ; H_{0}^{1}(\Omega)\right),
\end{aligned}
$$

and they are solutions of the problem $(* *)$ in $Q$. 
Proof of theorem 2. We consider $\left\{w_{j}\right\}, j=1,2, \ldots$, a Hilbertien basis in $\left[H_{0}^{1}(\Omega) \cap H^{2}(\Omega)\right]^{n}$. We represent by $V_{m}=\left[w_{1}, \ldots, w_{m}\right]$ the subspace generate by the vectors $w_{1}, \ldots, w_{m}$. We find $v_{m}(t)$ and $\phi_{m}(t)$ in $V_{m}$, solutions of the following system of ordinary differential equations:

$$
\begin{aligned}
& \left(v_{m}^{\prime \prime}, w\right)+a\left(t, v_{m}, w\right)-\left(\frac{(\lambda+\mu)}{K^{2}} \nabla d i v v_{m}, w\right)+\frac{\alpha}{K}\left(\nabla \phi_{m}, w\right)+\left(b_{i} \frac{\partial v_{m}^{\prime}}{\partial y_{i}}, w\right) \\
& +\left(c_{i} \frac{\partial v_{m}}{\partial y_{i}}, w\right)=0 \quad \forall w \in V_{m} . \\
& \left(\phi_{m}^{\prime}, w\right)+\frac{1}{K^{2}}\left(\nabla \phi_{m}, \nabla w\right)+\left(\frac{\beta}{K} d i v v_{m}^{\prime}, w\right)+\left(d_{i} \frac{\partial \phi_{m}}{\partial y_{i}}, w\right) \\
& +\left(\frac{\beta K^{\prime}}{K^{2}} \operatorname{div}_{m}, w\right)+\left(e_{i} \frac{\partial^{2} v_{j m}}{\partial y_{j} \partial y_{i}}, w\right)=0, \forall w \in V_{m} . \\
& v_{m}(0)=v_{0 m} \rightarrow v_{0} \text { in }\left[H_{0}^{1}(\Omega) \cap H^{2}(\Omega)\right]^{n} \\
& v_{m}^{\prime}(0)=v_{1 m} \rightarrow v_{1} \text { in }\left[H_{0}^{1}(\Omega)\right]^{n} \\
& \phi_{m}(0)=\phi_{0 m} \rightarrow \phi_{0} \text { in } H_{0}^{1}(\Omega) \cap H^{2}(\Omega) .
\end{aligned}
$$

First Estimate

Taking $w=v_{m}^{\prime}$ in (1) and $w=\phi_{m}$ in (2), we have:

$$
\begin{aligned}
& \frac{1}{2} \frac{d}{d t}\left|v_{m}^{\prime}\right|^{2}+\frac{1}{2} \frac{d}{d t} a\left(t, v_{m}, v_{m}\right)-\frac{1}{2} a^{\prime}\left(t, v_{m}, v_{m}\right)-\left(\frac{(\lambda+\mu)}{K^{2}} \nabla d i v v_{m}, v_{m}^{\prime}\right) \\
& +\frac{\alpha}{K}\left(\nabla \phi_{m}, v_{m}^{\prime}\right)+\left(b_{i} \frac{\partial v_{m}^{\prime}}{\partial y_{i}}, v_{m}^{\prime}\right)+\left(c_{i} \frac{\partial v_{m}^{\prime}}{\partial y_{i}}, v_{m}^{\prime}\right)=0 . \\
& \frac{1}{2} \frac{d}{d t}\left|\phi_{m}\right|^{2}+\frac{1}{K^{2}}\left\|\phi_{m}\right\|^{2}+\left(\frac{\beta}{K} \operatorname{div} v_{m}^{\prime}, \phi_{m}\right)+\left(d_{i} \frac{\partial \phi_{m}}{\partial y_{i}}, \phi_{m}\right) \\
& +\left(\frac{\beta K^{\prime}}{K^{2}} \text { div } \quad v_{m}, \phi_{m}\right)+\left(e_{i} \frac{\partial^{2} v_{j m}}{\partial y_{j} \partial y_{i}}, \phi_{m}\right)=0 .
\end{aligned}
$$


Developing some terms of (3) and (4), we obtain:

$$
\begin{aligned}
& -\left(\frac{(\lambda+\mu)}{K^{2}} \nabla \operatorname{div} v_{m}, v_{m}^{\prime}\right)=\frac{(\lambda+\mu)}{K^{2}}\left(\operatorname{div} v_{m}, \operatorname{div} v_{m}^{\prime}\right. \\
& =\frac{1}{2} \frac{d}{d t}\left(\frac{(\lambda+\mu)}{K^{2}}\left|\operatorname{div} v_{m}\right|^{2}\right)+\frac{(\lambda+\mu) K^{\prime}}{K^{3}}\left|\operatorname{div} v_{m}\right|^{2} \\
& \leq \frac{1}{2} \frac{d}{d t}\left(\frac{(\lambda+\mu)}{K^{2}}\left|\operatorname{div} v_{m}\right|^{2}\right)+c_{1}\left\|v_{m}\right\|^{2} \\
& \frac{\alpha}{K}\left(\nabla \phi_{m}, v_{m}^{\prime}\right)=\frac{\alpha}{K} \int_{\Omega} \nabla \phi_{m} v_{m}^{\prime} d y \\
& -\frac{\alpha}{K} \int_{\Omega} \phi_{m} \text { div } v_{m}^{\prime} d y=-\left(\frac{\alpha}{K} \operatorname{div} v_{m}^{\prime}, \phi_{m}\right)
\end{aligned}
$$$$
\left|a^{\prime}\left(t, v_{m}, v_{m}\right)\right| \leq c_{2}\left\|v_{m}\right\|^{2}
$$

$$
\begin{aligned}
& \left|\left(b_{i} \frac{\partial v_{m}^{\prime}}{\partial y_{i}}, v_{m}^{\prime}\right)\right|=\left|\frac{1}{2} \int_{2} b_{i} \frac{\partial\left|v_{m}^{\prime}\right|^{2}}{\partial y_{i}} d y\right| \\
& =\left.\frac{1}{2}\left|-\int_{2} \frac{\partial b_{i}}{\partial y_{i}}\right| v_{m}^{\prime}\right|^{2} d y+\int_{i}\left|b_{m}^{\prime}\right|^{2} \eta_{i} d \Gamma \mid \\
& \leq c_{3}\left|v_{m}^{\prime}\right|^{2} \\
& \left|\left(c_{i} \frac{\partial v_{m}}{\partial y_{i}}, v^{\prime}{ }_{m}\right)\right| \leq c_{4}\left(|| v_{m} \|^{2}+\left|v_{m}^{\prime}\right|^{2}\right) \\
& \left|\left(d_{i} \frac{\partial \phi_{m}}{\partial y_{i}}, \phi_{m}\right)\right|=\left.\frac{1}{2}\left|\int_{\Omega} d_{i} \frac{\partial}{\partial y_{i}}\right| \phi_{m}\right|^{2} d y \mid \\
& =\left.\left|-\frac{1}{2} \int_{\Omega} \frac{\partial d_{i}}{\partial y_{i}}\right| \phi_{m}\right|^{2} d y+\left.\frac{1}{2} \int_{\Gamma}\left|\phi_{m}\right|^{2} d_{i} \eta_{i} d \Gamma\left|\leq c_{5}\right| \phi_{m}\right|^{2}
\end{aligned}
$$

27 


$$
\begin{aligned}
& \left|\left(\frac{\beta K^{\prime}}{K^{2}} d i v v_{m}, \phi_{m}\right)\right| \leq c_{6}\left(\left\|v_{m}\right\|^{2}+\left|\phi_{m}\right|^{2}\right) \\
& \left(e_{i} \frac{\partial^{2} v_{m j}}{\partial y_{j} \partial y_{i}}, \phi_{m}\right)=\int_{\Omega} \phi_{m} \vec{e} \cdot \nabla\left(\operatorname{div} v_{m}\right) d y \\
& =-\int_{2} \operatorname{div}\left(\phi_{m} \vec{e}\right) \cdot \operatorname{div} v_{m} d y+\int_{\phi_{m}} \vec{e} \cdot \operatorname{div} v_{m} d \Gamma \\
& =-\int_{2} d i v\left(\phi_{m} \vec{e}\right) \cdot d i v v_{m} d y \text { where } \vec{e}=\left(e_{1}, \ldots, e_{n}\right) . \text { Thus, } \\
& \left|\left(e_{i} \frac{\partial^{2} v_{m j}}{\partial y_{j} \partial y_{i}}, \phi_{m}\right)\right| \leq \frac{1}{2 K^{2}}\left\|\phi_{m}\right\|^{2} .
\end{aligned}
$$

From (3) - (12), we have:

$$
\begin{aligned}
& \frac{1}{2} \frac{d}{d t}\left\{\frac{\beta}{\alpha}\left|v_{m}^{\prime}\right|^{2}+\frac{\beta}{\alpha} a\left(t, v_{m}, v_{m}\right)+\frac{\beta}{\alpha}\left(\frac{\lambda+\mu}{K^{2}}\right) d i v\left|v_{m}\right|^{2}+\left|\phi_{m}\right|^{2}\right\} \\
& +\frac{1}{2 K^{2}}\left\|\phi_{m}\right\|^{2} \leq c_{8}\left(\left\|v_{m}\right\|^{2}+\left|v_{m}^{\prime}\right|^{2}+\left|\phi_{m}\right|^{2}\right)
\end{aligned}
$$

Integrating (13) on $[0, t$ [ and applying Gronwall inequality, we conclude that there is a positive constant $C$ independent of $m$ and $t$, such that

$$
\begin{aligned}
& \frac{\beta}{\alpha}\left|v_{m}^{\prime}\right|^{2}+\frac{\beta}{\alpha} a\left(t, v_{m}, v_{m}\right) \frac{\beta}{\alpha}\left(\frac{\lambda+\mu}{K^{2}}\right) d i v\left|v_{m}\right|^{2} \\
& +\left|\phi_{m}\right|^{2}+\frac{1}{2 K^{2}} \int_{0}^{T}\left\|\phi_{m}\right\|^{2} d t \leq C,
\end{aligned}
$$




\section{Second Estimate}

Diferentiating (1) with respect to $t$ and taking $w=v_{m}^{\prime \prime}$, we obtain:

$$
\begin{aligned}
\left(v_{m}^{\prime \prime}, v_{m}^{\prime \prime}\right) & +a\left(t, v_{m}^{\prime}, v_{m}^{\prime \prime}\right)+a\left(t, v_{m}, v_{m}^{\prime \prime}\right)-\left(\frac{(\lambda+\mu)}{K^{2}} \nabla \operatorname{div} v_{m}^{\prime}, v_{m}^{\prime \prime}\right) \\
& +\frac{2(\lambda+\mu) K^{\prime}}{K^{3}}\left(\nabla \operatorname{div} v_{m}, v_{m}^{\prime \prime}\right)+\frac{\alpha}{K}\left(\nabla \phi_{m}, v_{m}^{\prime \prime}\right) \\
& +\left(b_{i} \frac{\partial v_{m}^{\prime \prime}}{\partial y_{i}}, v_{m}^{\prime \prime}\right)+\left(b_{i} \frac{\partial v_{m}}{\partial y_{i}}, v_{m}^{\prime \prime}\right)+\left(c_{i} \frac{\partial v_{m}^{\prime}}{\partial y_{i}}, v_{m}^{\prime \prime}\right)+\left(c_{i}^{\prime} \frac{\partial v_{m}}{\partial y_{i}}, v_{m}^{\prime \prime}\right)=0
\end{aligned}
$$

Diferentiating (2) with respect to $t$ and taking $w=\phi_{m}^{\prime}$, we have:

$$
\begin{aligned}
& \left(\phi_{m}^{\prime \prime}, \phi_{m}^{\prime}\right)+\frac{1}{K^{2}}\left|\nabla \phi_{m}^{\prime}\right| 2-\frac{2 K^{\prime}}{K^{3}}\left(\nabla \phi_{m}, \nabla \phi_{m}^{\prime}\right)+\left(\frac{\beta}{K} \operatorname{div} v_{m}^{\prime \prime}, \phi_{m}^{\prime}\right) \\
& +\left(d_{i} \frac{\partial \phi_{m}^{\prime}}{\partial y_{i}}, \phi_{m}^{\prime}\right)+\left(d_{i} \frac{\partial \phi_{m}}{\partial y_{i}}, \phi_{m}^{\prime}\right)+\left(\left(\frac{\beta K^{\prime}}{K^{2}}\right)^{\prime} \operatorname{div} v_{m}, \phi_{m}^{\prime}\right) \\
& +\left(e_{i} \frac{\partial^{2} v_{j m}}{\partial y_{i} \partial y_{i}}, \phi_{m}^{\prime}\right)+\left(e_{i}^{\prime} \frac{\partial^{2} v_{j m}}{\partial y_{i} \partial y_{i}}, \phi_{m}^{\prime}\right)=0 .
\end{aligned}
$$

Developing term terms of (15) and (16), we get:

$$
\begin{aligned}
& a^{\prime}\left(t, v_{m}, v_{m}^{\prime \prime}\right)=\int_{\Omega} a_{i j}^{\prime} \frac{\partial v_{m}}{\partial y_{i}} \frac{\partial v_{m}^{\prime \prime}}{\partial y_{i}} d y \\
& =\frac{d}{d t} \int_{\Omega} a_{i j}^{\prime} \frac{\partial v_{m}}{\partial y_{i}} \frac{\partial v_{m}^{\prime}}{\partial y_{i}} d y-\int_{\Omega} a_{i j}^{\prime \prime} \frac{\partial v_{m}}{\partial y_{i}} \frac{\partial v_{m}^{\prime}}{\partial y_{i}} d y-\int_{\Omega} a_{i j}^{\prime} \frac{\partial v_{m}^{\prime}}{\partial y_{i}} \frac{\partial v_{m}^{\prime}}{\partial y_{i}} d y \\
& \frac{2(\lambda+\mu) K^{\prime}}{K^{3}}\left(\nabla \operatorname{div} v_{m}, v_{m}^{\prime \prime}\right)=\frac{2(\lambda+\mu) K^{\prime}}{K^{3}} \int_{\Omega} \operatorname{div} v_{m} \operatorname{div} v_{m}^{\prime \prime} d y \\
& -\int_{\Omega} \frac{2(\lambda+\mu) K^{\prime}}{K^{3}}\left|\operatorname{div} v_{m}^{\prime}\right|^{2} d y .
\end{aligned}
$$




$$
\begin{aligned}
& \frac{-2 K^{\prime}}{K^{3}}\left(\nabla \phi_{m}, \nabla \phi_{m}^{\prime}\right)=\frac{d}{d t}\left(\frac{-K^{\prime}}{K^{3}}\left\|\phi_{m}\right\|^{2}\right)+\left(\frac{K^{\prime}}{K^{3}}\right)\left\|\phi_{m}\right\|^{2} \\
& \frac{-\alpha K^{\prime}}{K^{2}} \int_{2} \nabla \phi_{m} v_{m}^{\prime \prime} d y \leq C_{9}\left|v_{m}^{\prime \prime}\right|^{2}+\frac{1}{4 K^{2}}\left\|\phi_{m}\right\|^{2} \\
& \left(e_{i}^{\prime} \frac{\partial^{2} v_{j m}}{\partial y_{j} \partial y_{i}}, \phi^{\prime}{ }_{m}\right)=\int_{2} e^{\prime}{ }_{i} \frac{\partial}{\partial y_{j}}\left(\frac{\partial v_{j m}}{\partial y_{i}}\right) \phi_{m}^{\prime} d y=\int_{2} e_{i}^{\prime} d i v \frac{\partial v_{m}}{\partial y_{i}} \phi_{m}^{\prime} d y \\
& \quad=-\int_{\Omega} \frac{\partial}{\partial y_{i}}\left(e^{\prime}{ }_{i} \phi_{m}^{\prime}\right) d i v v_{m} d y+\int_{\Gamma} d i v v_{m} e_{i}^{\prime} \phi_{m}^{\prime} \eta_{i} d \Gamma \\
& \quad=-\int_{\Omega} \frac{\partial e_{i}^{\prime}}{\partial y_{i}} \phi_{m}^{\prime} d i v v_{m} d y-\int_{\Omega} e_{i}^{\prime} \frac{\partial \phi_{m}^{\prime}}{\partial y_{i}} \operatorname{div} v_{m} d y \\
& \leq c_{10}\left(\left|\phi_{m}^{\prime}\right|^{2}+\left\|v_{m}\right\|^{2}\right)+\frac{1}{4 K^{2}}\left\|\phi_{m}^{\prime}\right\|^{2} .
\end{aligned}
$$

From (15)-(21), we obtain:

$$
\begin{gathered}
\frac{1}{2} \frac{d}{d t}\left\{\frac{\beta}{\alpha}\left|v_{m}^{\prime \prime}\right|^{2}+\frac{\beta}{\alpha} a\left(t, v_{m}^{\prime}, v_{m}^{\prime}\right)+\frac{\beta}{\alpha} \frac{(\lambda+\mu)}{K^{2}}\left|\operatorname{div} v_{m}^{\prime}\right|^{2}+\left|\phi_{m}^{\prime}\right|^{2}\right. \\
\left.+\frac{\beta}{\alpha} \int_{\Omega} a_{i j}^{\prime} \frac{\partial v_{m}}{\partial y_{i}} \frac{\partial v_{m}^{\prime}}{\partial y_{j}} d y+\frac{\beta}{\alpha} \int_{\Omega} \frac{2(\lambda+\mu) K^{\prime}}{K^{3}} d i v v_{m} \operatorname{div} v_{m}^{\prime} d y-\frac{K^{\prime}}{K^{3}}\left\|\phi_{m}\right\|\right\} \\
+\frac{1}{4 K^{2}}\left\|\phi_{m}^{\prime}\right\| \|^{2} \leq c\left[\left\|v_{m}^{\prime}\right\|\left\|^{2}+\left|v_{m}^{\prime \prime}\right|^{2}+\left|\phi_{m}^{\prime}\right|^{2}+\right\| v_{m}\left\|^{2}+\right\| \phi_{m} \|^{2}\right]
\end{gathered}
$$

Integrating (22) on $[0, t[$, and applying Gronwall inequality, we deduce:

$$
\begin{aligned}
& \frac{\beta}{\alpha}\left|v_{m}^{\prime \prime}\right|^{2}+\frac{\beta}{2 \alpha} \rho_{0}\left\|v_{m}^{\prime}\right\|^{2}+\frac{\beta}{\alpha} \frac{(\lambda+\mu)}{K^{2}}\left|\operatorname{div} v_{m}^{\prime}\right|^{2}+\left|\phi_{m}^{\prime}\right|^{2} \\
& +\int_{0}^{T} \frac{1}{8 K^{2}}\left\|\phi_{m}^{\prime}\right\|{ }^{2} d t \leq C
\end{aligned}
$$

30 
From (14) and (23), it follows that:

$$
\mid \begin{aligned}
& v_{m}^{\prime \prime} \rightarrow v^{\prime \prime} \quad \text { weak star in } L^{\infty}\left(0, T ;\left[L^{2}(\Omega)\right]^{n}\right) \\
& v_{m} \rightarrow v \quad \text { weak star in } L^{\infty}\left(0, T ;\left[H_{0}^{1}(\Omega)\right]^{n}\right) \\
& \frac{1}{K} \phi_{m} \rightarrow \frac{1}{K} \phi \quad \text { weak star in } L^{\infty}\left(0, T ; H_{0}^{1}(\Omega)\right) \\
& \phi_{m}^{\prime} \rightarrow \phi^{\prime} \quad \text { weak star in } L^{2}\left(0, T ; H_{0}^{1}(\Omega)\right)
\end{aligned}
$$

From (24) taking limits in (1) and (2), we have the proof of the theorem 2.

\section{Proof of theorem 1.}

We set

$$
\begin{aligned}
& { }_{0}(y)=u_{0}(K(0) y), \quad \phi_{0}(y)=\theta_{0}(K(0) y), \\
& { }_{1}(y)=K^{\prime}(0) \nabla u_{0}(K(0) y) \cdot y+u_{1}(K(0) y)
\end{aligned}
$$

that satisfy the hypothesis of theorem 2 .

Therefore, there exist $\quad, \phi$ solutions of the problem (**). Using and $\phi$ we construct $u$ and given by:

The regularity of $u$ and can be obtained from,$\phi$ and the hypothesis H1). We also verify the following identities:

$$
\begin{aligned}
& \alpha \nabla \theta=\frac{\alpha}{K} \nabla \phi \\
& (\lambda+\mu) \nabla \operatorname{div} u=\frac{\lambda+\mu}{K^{2}} \nabla \operatorname{div} v \\
& \mu \Delta u=\frac{\mu}{K^{2}} \Delta v \\
& u^{\prime \prime}=v^{\prime \prime}-\frac{\partial}{\partial y_{j}}\left(a_{i j} \frac{\partial v}{\partial y_{i}}\right)+\frac{\mu}{K^{2}} \Delta v+b_{i} \frac{\partial v^{\prime}}{\partial y_{i}}+c_{i} \frac{\partial v}{\partial y_{i}}
\end{aligned}
$$




$$
\begin{aligned}
& \Delta \theta=\frac{1}{K^{2}} \Delta \phi \\
& \beta \text { div } u^{\prime}=\frac{\beta}{K} \operatorname{div} v^{\prime}+\frac{\beta K^{\prime}}{K^{2}} \operatorname{div} v+e_{i} \frac{\partial^{2} v_{j}}{\partial y_{j} \partial y_{i}} \\
& \theta^{\prime}=\phi^{\prime}+d_{i} \frac{\partial \phi}{\partial y_{i}}
\end{aligned}
$$

from (27)-(33), using the fact that $(v, \phi)$ satisfies $(* *)$, we deduce that $(u, \theta)$ satisfies $(*)$, and hence the theorem 1 is completely proved.

\section{REFERENCES}

1. Barreto, R.K. and Rivera, J.E.M.; Existence and exponential decay in nonlinear thermoelasticity. Nonlinear Analysis, Theory Methods and Applications, vol. 31, n 1/2 (1998), pp. 149-162.

2. Caldas, C.S.; Limaco, J and Barreto, R.K.; Linear Thermoelastic system in non-cylindrical domains, Funckcialaj Ekvacioj, 42 (1999), pp. 115-127.

3. Dafermos, C.M.; On the existence and the asymptotic stability of solution to the equations of linear thermoelasticity, Arch. Rational Mech. Anal., vol. 29 (1968), pp. 241-271.

4. Henry, D.; Lopes, O. And Parisinotto, A.; Linear Thermoelasticity: asymptotic stability and essential spectrum (unpublished manuscript).

5. Lagnese, J.E.; The reachability problem for thermoelastic plates, Arch. Rat. Mech. Anal. 112, (1990). Pp. 223-267.

6. Rivera, J.E.M.; Exponential decay in thermoelasticity, Funkcialaj Ekvacioj, 35 (1992), pp. 19-30. 